\title{
Development of a Novel Robot-Assisted Orthopaedic System Designed for Total Knee Arthroplasty
}

\author{
Naohiko Sugita $^{1}$, Shin'ichi Warisawa ${ }^{1}$, Mamoru Mitsuishi ${ }^{1}$, Masahiko Suzuki², $^{2}$ \\ Hideshige Moriya $^{2}$, and Koichi Kuramoto ${ }^{3}$ \\ 1 University of the Tokyo, 7-3-1 Hongo Bunkyo-ku Tokyo, Japan \\ ${ }^{2}$ Chiba University, 1-8-1 Inohana Chuo-ku Chiba, Japan \\ 3 Nakashima Propeller Co.,Ltd., 688-1 Jotokitakata Okayama, Japan
}

\begin{abstract}
Recently, the number of robot-assisted orthopaedic system which supports surgeon has increased. As one of the background, it is considered the difference between plan and result of the bone cutting is varied because the technique to resect bone is based on the ability of individual surgeon. And authors developed a robot to resect bone which has 9 degree of freedom, and as the result, the accuracy of bone cutting improved. Moreover, we developed a total operation system including pre-operative planning system and intra-operative assist system.
\end{abstract}

\section{Introduction}

In the total knee arthroplasty(TKA), the accuracy to set prosthesis affects the inferior limb direction after operation. If the prosthesis is not properly fixed, the post-operative pain, life time reduction of the prosthesis and organization necrosis caused by the abrasion powder may occur. Therefore, it is hoped to improve the accuracy of prosthesis position, and robots to support the bone resection have been introduced rapidly.

In this paper, a developed bone cutting robot which has multiple degrees of freedom is discussed. The movements of this equipment during the bone resection are restricted within the cut plane, and this function is realized by 2 axes for translation and 1 axis for rotation to avoid soft tissue like ligaments. High accuracy and safety are acquired by this technology. And this robot-assisted total knee arthroplasty system has a feature that surgeon can intervene and modify pre-operative plan during operation. It is difficult to judge the state of soft tissue clearly before the operation, and this system allows surgeon to intervene into the system interactively and to modify the pre-operative plan after incising the cutis. Finally, results of the improved bone cutting accuracy with some experiments are presented.

\section{Related Work}

Computer-assisted orthopaedic system is classified into two categories mainly. [1] One is what guides the portion to resect the bone and the position of prosthesis using navigation system with or without computer tomography .(for 
example,[2],etc) The other is what supports surgeon with a robot to cut the bone adding into navigation functions. 3]- 5]

This study belongs to the latter. The main robot-assisted orthopaedic systems for lower limb are ROBODOC 3],CASPAR 4],ACROBOT[5]. This system has two main features compared to other systems. First, cartilage and soft tissue can be considered because surgeon can change the pre-operative plan and modify the tool path for the bone resection. And a minimum invasive registration method without any pins is adopted.

On the other hand, some papers report the comparison results in the case of robot-assisted operation and manual operation. 6]-7] Honl,et al[6] reports that they did THA operation using ROBODOC more than 50 cases, and they compared the accuracy, the condition after operation, time of operation and etc to the manual operation. As the result, it is recognized that the operation with the robot gives the improvement of the accuracy.

\section{Pre-operative Assisting System}

Overview of the application for total knee arthroplasty we developed is shown in Fig.1. Cross section image, predicted image at any location and projected image are presented on the display, and surgeon decides type and position of prosthesis, and tool path for the bone resection is generated automatically. The determination of prosthesis position is based on the anatomical distinction. Surgeon can decide the position of artificial knee joint considering the total shape of the limb by indicating some points on the display according to the guide of application.

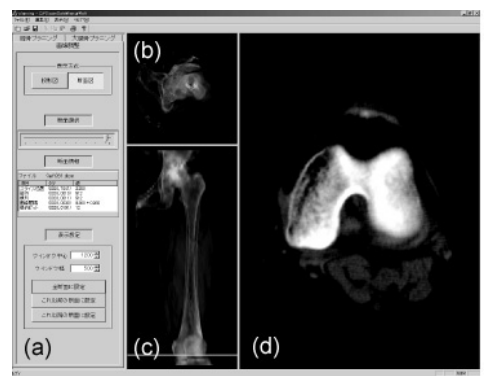

Operation window

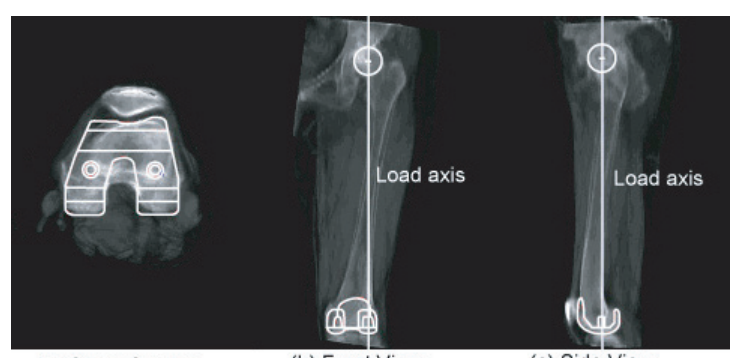

(a) Cross Section

(b) Front View

(c) Side View

\section{Configuration of prosthesis}

Fig. 1. Operation Planning Software: Operation window (a)operation window (b)cross section window (c)front view window(d)main window and Configuration of prosthesis

Then, tool path for the bone resection is generated from the information of the planned prosthesis position. First, the area which tool occupies is defined automatically, and surgeon modifies the area to assure safety. And tool path on the cut plane is generated. Coordinates for each cut plane is defined so that 
plane and area for the bone resection are within the range of $\mathrm{U}, \mathrm{V}, \mathrm{W}, \mathrm{A}, \mathrm{B}$ and $\mathrm{C}$ axes in the bone cutting robot (Fig 2)

\section{Bone Cutting Robot}

Overview of the bone cutting robot which has 9 degree of freedom(d.o.f) is shown in Fig 2. The size of robot is $810 \mathrm{~mm} \times 1500 \mathrm{~mm} \times 2050 \mathrm{~mm}$, and the weight is approximately $900 \mathrm{~kg}$.

\subsection{Requirements for Bone Cutting Robot}

The requirements for the bone cutting robot are as follows.

- Surface roughness and angle error accuracy between adjacent cut planes It is required that the surface roughness and angle error between planes become smaller. And to realize the bone cutting with the high accuracy, a solid structure is desired.

- Assurance of safety during bone resection

Monitoring of the abnormal state with cutting force, mechanical safety with fail-safe mechanism and emergency button are equipped for this robot. And work space for surgeon should be also considered.

\subsection{Characteristics of Mechanism}

As mechanical and structural features, the following points are listed.

- Tip position of tool is fixed at the center of 3 rotation d.o.f.

When the posture of tool is changed, the tip of tool does not move and the safety is kept.

- Movement of tool during resection is restricted within the cut plane Axes for bone cutting are defined in advance and the cutting tool is restricted within the cut plane mechanically. And the improvements of safety and cutting accuracy are realized.

- Definition and clarification of role for each axis

The behavior of robot can be predicted by clarifying the role for each axis, and the motion control like modification of tool path becomes easier.

- Force sensor is equipped

It is possible to monitor the cutting status by measuring the cutting force.

- Fail-safe mechanism is equipped

Patient and robot can be protected mechanically.

\subsection{Strategy for Layout}

The motion of the robot can be predicted by clarifying the role for each axis, and the control like the modification of tool path becomes easier. To resect the bone precisely, axes for approaching to the operation area and axes for resecting the bone are separated respectively. Axes for rough position (X,Y and Z) are located 
around the base, and axes for fine position (3 rotational and 3 translational axes) are arranged near the tool.

Axes for bone cutting are defined in advance and the cutting tool is restricted within the cut plane mechanically. And the improvements of safety and cutting accuracy are realized.

Axes for the bone cutting is assigned to realize 2 dimensional motion, and the cutting tool is restricted within the plane. More precise cutting is expected by this simple process. Plane inclination is decided by 2 rotational axes (A and $\mathrm{B})$, and 1 translational axis (W) controls the position in radius direction. To cut the plane, 2 translational axes $(\mathrm{U}$ and $\mathrm{V})$ and 1 rotational axis $(\mathrm{C})$ to rotate the tool are used.

When the posture of tool is changed, the tip of tool is fixed at the center of 3 rotational axes, and the safety is kept.

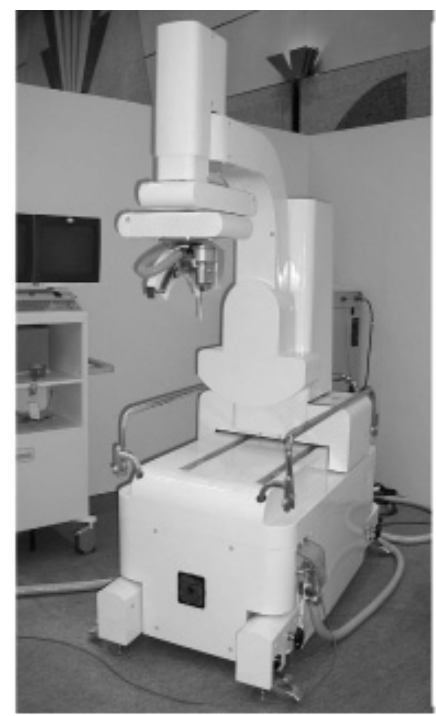

(a) Bone cutting machine

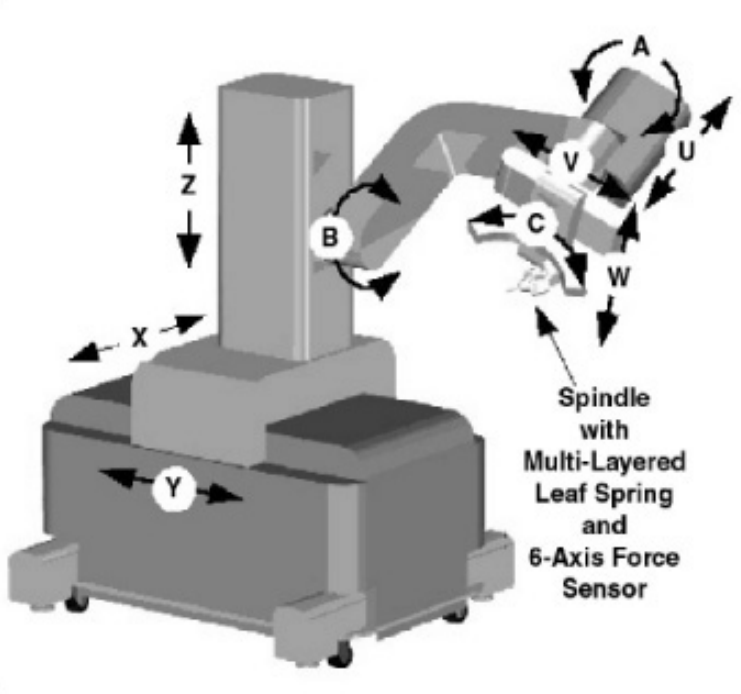

(b) Configuration of axes

Fig. 2. Overview of bone cutting machine: (a)Bone cutting machine (b) Configuration of axes

\subsection{Controller}

The controller for the bone cutting system consists of a PC for the user interface and a real-time controller. Both computers are connected with LAN. Real time Linux is adopted as a operating system. The controller accepts a command from user interface PC by socket communication on TCP/IP even while it is controlling the cutting machine. The user interface on PC is shown in Fig 3 . 


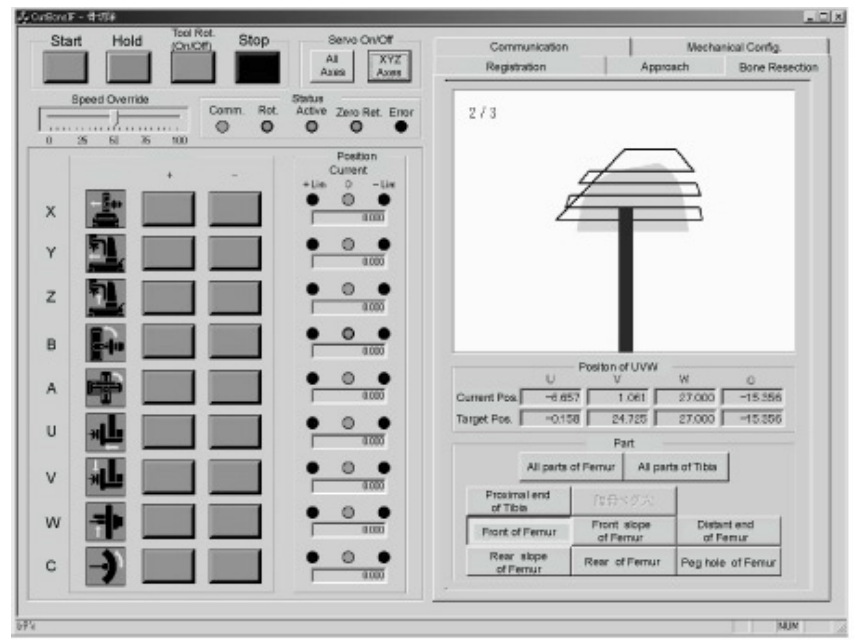

Fig. 3. Operation window for surgeon as user interface

\section{Intra-operative Assist System}

A registration method which does not need to incise the cutis any more and to use marker pins is introduced. In the registration, the characteristic points which does not depend on individual surgeon are defined as bony landmarks, and the accuracy to probe can be improved. Then, the points indicated on the user interface are identified using the tool part of the robot. The position of the patient on the bed is recognized by the registration, and the approach point for the bone resection and the posture of the robot are decided.

The tool path generated in pre-operative system is based on the image of CT, and it is difficult to consider soft tissue in advance. Therefore, the pre-operative plan can be changed to restrict the area which the tool invades during the operation, and the risk to damage the ligaments, nerves and vessels is lowered. In detail, $\mathrm{C}$ axis in Fig 2 is used to rotate the tool posture while cutting and to avoid the hazardous area. Surgeon can configure allowable area which the tool invades, and the tool path is recalculated by the intra-operative system. Finally, the surgeon confirms the represented path.

\section{System Evaluation}

\subsection{Measurement of Surface Roughness and the Angle Accuracy}

5 surfaces of model bone for the artificial knee joint were cut using the developed system. The accuracy of the cut surface was compared with that obtained by the conventional method. Each surface was measured by a CMM. The number of sampled points was from 200 to 500, depending on the plane. An approximate 
plane was calculated using the sampled point data for each surface. The surface roughness was obtained by calculating the variance of the sampled points from the approximate plane. Fig 4 shows the experimental results. The angle between the desired angle and the experimentally obtained angle is shown in Fig 4. The surface roughness and angle accuracy were improved from 200 to $33.2 \mu \mathrm{m}$ and 4.49 to 0.125 deg., respectively.
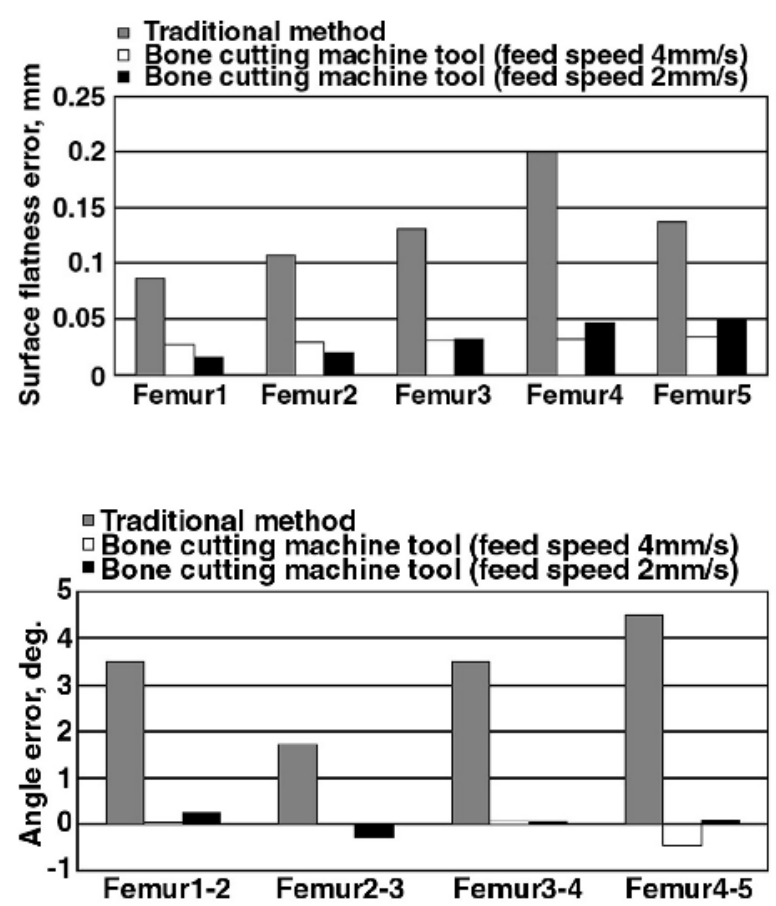

Fig. 4. Comparison of surface roughness(above) and angle accuracy(below)

\subsection{Cadaveric Bone Cutting Experiment}

Using the developed system, a experiment of the bone cutting was done with cadaver. Regarding the angle of planes to fix the prosthesis, the difference between plan and result was evaluated.

Fig 5 shows the look of this experiment. As the cut plane of femur, two are in front, one in distal end and one in rear. And each plane is connected with curved surface. When the planes are cut, two planes in front are treated as one and each curved surface is approximated as a plane. The evaluation of angle between planes is described in Fig 6 


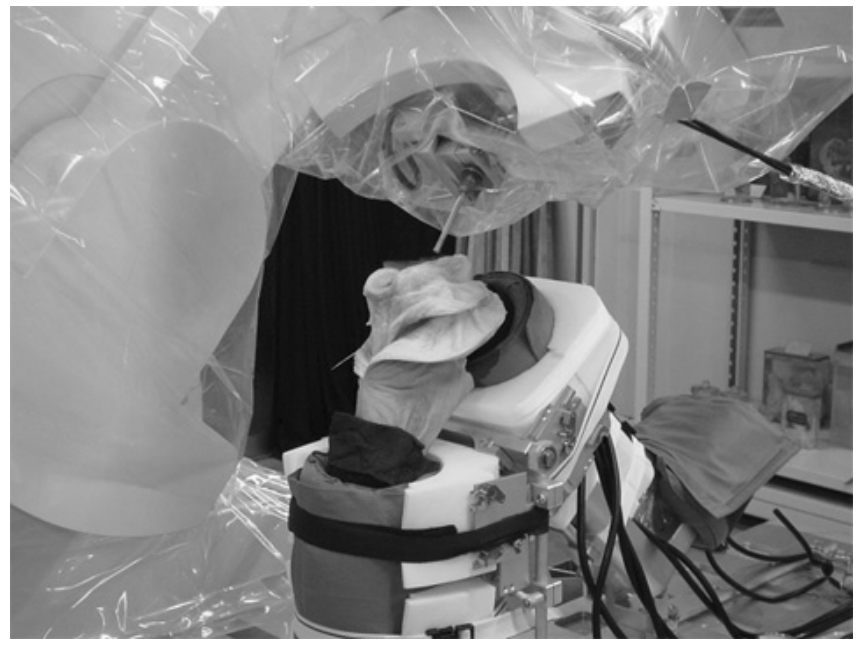

Fig. 5. Cadaveric bone cutting experiment

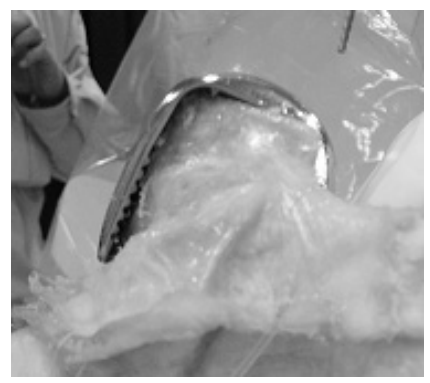

(a) Set of prosthesis

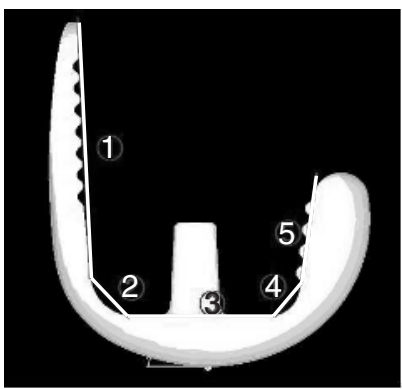

(c) Joint planes

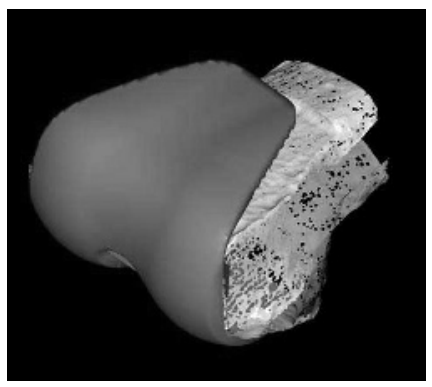

(b) CAD analyzation

\begin{tabular}{|c|c|c|c|}
\hline Angle & Plan & Result & Diff. \\
\hline $1-3$ & $93.3^{\circ}$ & $93.8^{\circ}$ & $0.5^{\circ}$ \\
\hline $3-5$ & $98.0^{\circ}$ & $99.0^{\circ}$ & $1.0^{\circ}$ \\
\hline $1-3$ & $11.3^{\circ}$ & $12.8^{\circ}$ & $1.5^{\circ}$ \\
\hline
\end{tabular}

(d) Result

Fig. 6. Evaluation of angles between cut planes 
With the current technique the error of maximum 4.5 degree may occur, and this experiment showed the error of 1.5 degree at most. From the result, the system is useful for more precise bone cutting.

\section{Conclusions}

In this paper, a robot-assisted orthopaedic system designed for total knee arthroplasty was described, and the surgeon can modify the pre-operative plan during the operation. And from the result of the experiment with model bone and cadaver, it becomes clear that surface roughness and angle difference between cut planes can be lower than the current operation technique.

\section{References}

1. DiGioia AM, Jaramaz B, Colgan BD: Computer Assisted Orthopaedic Surgery: Image Guided and Robotic Assistive Technologies. Clin. Orthop., 354:8-16, 1998

2. Stulberg SD, Loan P, et al: Computer-Assisted Navigation in Total Knee Replacement: Results of an Initial Experience in Thirty-Five Patients. J. Bone Joint Surg.,84-A (2002) S90-98

3. Mittelstadt BD, Kazanzides P, Zuhars J: The Evolution of a Surgical Robot from Prototype to Human Clinical Trial. Proc. Medical Robotics and Computer Assisted Surery (1994)

4. Peterman J, Kober R, Heinze P, Heekt P, Gotzen L: Implementation of the CASPAR System in the reconstruction of the ACL. CAOS/USA. (2000) 86-87

5. Delp SL, Stulbrg DS, David S, Davies B, Picard F, Leitner F: Computer Assisted Knee Replacement. Clin. Orthop. 354 (1998) 49-56

6. Honl M, Dierk O, Gauck C, Carrero V, Lampe F, Dries S, Quante M, Schwieger K, Hille E, Morlock MM: Comparison of Robotic-Assisted and Manual Implantation of a Primary Total Hip Replacement. J. Bone Joint Surg., 85-A (2003) 1470-1478.

7. Krismer M, Nogler M, Kaufmann CH, Ogon M: Revision of Femoral Component: Cement Removal by Robot vs. Manual Procedure. J.Bone Joint Surg. 83-B SI (2001) 80 